\title{
Les compétences discursives et génériques dans la traduction des contrats de droit civil français et polonais
}

\section{Discursive and generic competences in translating French and Polish civil law contracts}

\author{
Dorota Lajus \\ Uniwersytet Mikołaja Kopernika w Toruniu \\ dorotalajus@umk.pl
}

\begin{abstract}
In the process of legal translation, different rules for editing individual genres are an important issue arising from cultural differences. Therefore, translator's discursive and generic competences affect the quality of translation. Even if the knowledge of standards of the genre of target culture does not allow their direct application in the text of translation, a comparison of generic standards facilitates proper interpretation of the source text and editing the target text, thus allowing to achieve the intended communicative objective. The purpose of this article is to compare the legal discourse in highly routinised genres such as Polish and French contracts in the field of civil law. Based on the French school of discourse analysis, the study is designed to extract meanings which are not expressed directly, but are related to the function of a given text. Particular emphasis is placed on ways of expressing the performative nature of analysed genres and interpersonal relationships.
\end{abstract}

Keywords: contract, discursive competences, generic competences, legal discourse, genre, legal translation

La traduction juridique se confronte à de nombreux problèmes issus entre autres du caractère culturel du système de droit donné et des divergences entre les systèmes juridiques en présence. Ces différences culturelles se manifestent notamment dans les conventions ou les règles de rédaction des textes. C'est pourquoi l'activité traduisante met en œuvre les compétences discursives et génériques. En effet, même si la connaissance des normes du genre d'arrivée ne permet pas leur application directe, la comparaison des genres favorise une interprétation et une rédaction correctes des textes, et de ce fait elle permet d'atteindre le but communicatif visé. Les analyses discursives et génériques, par 
les moyens qu'elles donnent à la compréhension des divers genres de texte, aident le traducteur à se rendre compte des significations implicites des textes, qui dépendent en grande partie de la fonction du texte et de ses effets pragmatiques.

En s'appuyant sur l'école française de l'analyse du discours, le présent article a pour but de comparer la réalisation du discours juridique dans des genres hautement routinisés, tels que les contrats français et polonais relevant du droit civil.

Selon le modèle interprétatif de la traduction, cette dernière est considérée comme un acte de communication dont l'objet est un texte produit dans une situation de communication précise, par un émetteur précis et adressé à un destinataire précis pour accomplir un but communicatif déterminé. Par conséquent, pour comprendre le texte de départ et produire celui d'arrivée, le traducteur est obligé de disposer de compétences discursives. Pour cela, il peut puiser dans l'analyse du discours. Celle-ci met également en évidence l'importance des genres discursifs.

Plus un genre de texte est formalisé, plus l'interprétation adéquate d'un texte appartenant à ce genre dépend de la correction de sa rédaction, de la conformité aux normes génériques, ce qui dans le contexte de la traduction implique la nécessité de la connaissance du domaine non seulement du point de vue de l'objet mais aussi du point de vue discursif (Walkiewicz, 2013 : 9).

\section{DISCOURS, GENRE, COMPÉTENCES DISCURSIVES ET GÉNÉRIQUES}

Dans notre travail, nous comprenons le discours dans le sens de l'école française de l'analyse du discours et reprenons la distinction entre texte, genre et discours proposée par H. Grzmil-Tylutki (2007). La notion de discours permet de mettre l'accent sur les dimensions institutionnelles des activités langagières, sur le rituel socio-langagier dont le respect garantit la réussite d'un acte de langage. Le terme de discours embrasse un ensemble de relations intersubjectives et situationnelles institutionnalisées et les taxinomies des discours s'appuient sur les domaines, les thèmes et les fonctions pragmatiques. Le discours n'a pas d'existence dans l'abstrait, il se distribue toujours en genres qui revêtent des formes textuelles (Grzmil-Tylutki, 2007: 239-243). Le genre en revanche est compris ici comme un moyen de communication qui permet à des membres d'une communauté linguisique de produire et de comprendre des énoncés en fonction des conditions socio-culturelles et historiques. Il est formé d'un ensemble de textes concrets présentant des traits communs en ce qui 
concerne le but et les formes préférées. Il constitue un intermédiaire entre le discours et le texte. Les textes enfin sont des formes réelles, empiriques, constituant une matérialisation directe du genre et indirecte du discours (GrzmilTylutki, 2007 : 40-42, 232-242). Les compétences discursives et génériques font partie des compétences communicatives (Grzmil-Tylutki, 2007: 67). Elles définissent notre capacité à produire et comprendre des énoncés dans des situations concrètes, et répondent aux normes génériques d'une culture donnée.

En lisant les définitions du discours juridique, des textes de droit ou juridiques élaborées par les juristes, les traductologues et les jurilinguistes, on constate que les critères de définition sont proches, ils mettent en avant les relations interpersonnelles et la fonction du texte. La définition donnée par G. Cornu est basée sur la finalité: «Est juridique tout discours qui a pour objet la création ou la réalisation du droit» (Cornu, 2007: 22). Comme exemple de genre de texte juridique, on peut citer une loi dont l'auteur est le législateur et qui crée des normes concernant tous ou certains sujets de droit, un jugement d'un tribunal, un acte notarié créant une société, etc. Cependant, certains textes rédigés par des spécialistes et se référant au droit ne font pas naître des réalités juridiques mais commentent le droit et ses pratiques. D'autres textes émanent des non-initiés et engendrent des conséquences de droit. Le caractère juridique d'un texte résulte donc selon G. Cornu (2007) de son but, et non du sujet de communication, de la personnalité des locuteurs, du vocabulaire ou du style employés. Il distingue nettement le discours et le vocabulaire juridique, soulignant qu' «il peut y avoir discours juridique sans vocabulaire juridique » (Cornu, 2007 : 213). D'après cet auteur, cette distinction ne nie pas l'intérêt de l'analyse du vocabulaire ou de la syntaxe d'un texte juridique, elle change seulement le critère de juridicité du discours et élargit par conséquent la notion de discours juridique. Ainsi, on peut compter parmi les genres juridiques non seulement l'édiction d'une loi, le prononcé d'un jugement, l'établissement d'une convention, mais aussi par exemple le constat d'un dommage, la déclaration d'un témoin, la convocation d'un plaideur, l'avis d'un expert, etc. Nous n'évoquerons pas dans cet article le vaste sujet des taxinomies du discours juridique proposées par les linguistes, jurilinguistes ou juristes polonais et francophones.

\section{PRÉSENTATION DU CORPUS}

Dans ce travail, nous comparons la réalisation du discours juridique dans les genres hautement routinisés, à savoir dans les contrats choisis polonais et français relevant des droits civils des deux pays. Le contrat est dans le Code civil 
français défini comme « une convention par laquelle une ou plusieurs personnes s'obligent, envers une ou plusieurs autres, à donner, à faire ou à ne pas faire quelque chose » (Code civil, art. 1101). Il s'agit donc d'un acte juridique qui résulte de l'accord de deux ou plusieurs volontés et qui fait naître des obligations ou bien crée ou transfère un droit réel (voir Guillien, 2001 : 151; Fontaine, Cavalerie \& Hassenforder, 2000: 97). Cette définition est proche du terme umowa dans le droit polonais (Radwański, 2008).

Nous consacrons notre étude aux contrats relevant du droit civil, celui de l'individu, notamment les contrats de bail, de prêt, de prêt à usage, de donation, les statuts de société civile. Ils appartiennent aux genres de textes hautement stéréotypés et ces derniers ont été l'objet de recherches de R. Solova (2013), qui présente une étude sur la norme et la pratique dans la traduction certifiée de polonais en français et de français en polonais. Mais cette chercheuse a travaillé sur d'autres genres tels que les actes d'état civil, les diplômes d'études, les extraits de casier judiciaire, les contrats de travail. Les contrats et les statuts de société en tant que genres sont analysés chez A. Jopek-Bosiacka (2006), qui compare les écrits polonais et anglais et propose des solutions de traduction.

Ces textes sont des actes, ils produisent des effets juridiques tels que la création d'une société, la donation, la collation d'un pouvoir, etc. Les actes s'accomplissent par la prise de parole, par la prononciation de la volonté des signataires. Les mots, élevés au rang d'actes, acquièrent une importance particulière, ils sont ritualisés par des conventions, et sont plus ou moins figés. Il faudrait noter que le droit distingue deux acceptions du terme " acte ». La première est l'instrumentum, écrit indispensable à la validité ou à la preuve d'une situation juridique. La deuxième est celle de negotium, expression d'une volonté destinée à produire des effets de droit.

Parmi les écrits étudiés, il y a des actes sous seing privé, rédigés et signés par des particuliers sans la présence de tiers. D'autres contrats sont établis sous la forme authentique, c'est-à-dire qu'ils sont reçus par un officier public. A ce groupe appartiennent les actes notariés. Dans notre corpus, il y a des contrats unilatéraux, ne faisant naître de prestations qu'à la charge d'une seule partie, comme le contrat de donation. Outre ceci, il y a des contrats synallagmatiques ou bilatéraux, dans lesquels les contractants s'obligent réciproquement les uns envers les autres, ce qui est par exemple le cas du contrat de bail. Chacun de ces types d'acte doit respecter certaines conditions de forme et de contenu pour être valables c'est-à-dire pour produire des effets de droit.

Notre corpus est constitué de trois sortes de documents. Les premiers sont des documents établis réellement par les officiers publics ou les personnes privées, ils portent les signatures, les tampons et d'autres marques des personnes intervenant dans l'acte. Le deuxième groupe est constitué de formulaires 
officiels, tout faits, que l'on remplit à la main. Quant au troisième type de documents, il s'agit des modèles de textes juridiques préfabriqués par les juristes, adressés aux professionnels et aux particuliers.

Ces genres sont hautement stéréotypés en ce qui concerne leur forme, leur présentation graphique et leurs composants. Leur caractère conventionnel résulte non seulement de l'usage mais aussi des lois qui prévoient les formes nécessaires à la validité des actes, les éléments de contenu indispensables, etc. Le caractère solennel des documents est souligné par leur mise en page, les tampons, les signatures; leur structure est claire, bien accentuée avec des encadrements, des titres ou le numéro des articles, etc. (Antoniewska, 2005)

D'après J.-C. Gémar, le contrat est le genre de texte juridique qui est le plus obscur en apparence parce que le plus formalisé, technique et archaïque. Mais selon cet auteur, la technicité des contrats est plus apparente que réelle. Bien que la forme ou les tournures particulières à ce type de texte en troublent la lisibilité, la compréhension et la traduction de ces textes n'est pas plus difficile que celle d'une loi ou d'un jugement (Gémar, 1995 : 121).

La mise en page et la macrostructure ne font pas l'objet de cet article, nous analyserons les contrats en tant que genre au niveau micro, celui des éléments linguistiques qui deviennent une routine scénographique imposée par les relations intersubjectives et situationnelles et par un but pragmatique précis (Grzmil-Tylutki, 2007 : 116). Etant donné la dimension de cet article, celui-ci ne sera consacré qu'à certains aspects, à savoir aux moyens d'expression des relations intersubjectives et du caractère performatif de ces textes.

\section{COMPARAISON DES RELATIONS INTERSUBJECTVES DANS LES CONTRATS FRANÇAIS ET POLONAIS}

3.1. Le discours juridique se caractérise par un ton neutre et objectif, voire formel. L'un des traits qui tend à donner cet effet est l'impersonnalité. Ainsi, les contrats sont écrits à la troisième personne et le locuteur parle de lui-même comme s'il était un tiers. L'emploi de la non-personne produit un effet d'objectivation et contribue à donner de l'importance au locuteur. Les signataires des actes étudiés ne sont pas traités comme des personnes individuelles mais plutôt en fonction de leurs rôles. Même si un acte juridique est l'expression de la volonté des signataires, il se fait en application de la loi qui est générale, parle à la troisième personne et définit les comportements des personnes dans des situations déterminées : situation du bailleur, de l'employeur, etc. Ainsi, nous citons à titre d'exemple, deux formules introduisant des stipulations de contrats qui sont l'effet d'un accord entre les parties : 
Les soussignés déclarent ce qui suit...

Les parties conviennent ce qui suit...

En outre, comme nous pouvons le constater en observant ces exemples, les signataires des contrats, présentés dans le préambule, sont ensuite désignés par leur fonction : le bailleur, le locataire, le preneur, le créancier, le débiteur, le donateur, le donataire, le mandant, le mandataire, le prêteur, l'emprunteur, le propriétaire, l'associé, le soussigné, wynajmujący, najemca, wydzierżawiający, dzierżawca, wierzyciel, dłużnik, darczyńca, obdarowany, mocodawca, pełnomocnik, pożyczkodawca, pożyczkobiorca, użyczający, biorący do używania, właściciel, wspólnik, niżej podpisany, etc. Et ceci, à l'aide de formules telles que :

M.... ci-après dénommé le prêteur...

M... désigné ci-après indifféremment le requérant

M... qualifié ci-après comme étant le donateur

... zwany dalej Pożyczkodawca...

Il en est de même pour les notaires qui dans les préambules ou les clôtures parlent d'eux-mêmes à la troisième personne :

Me ..., notaire à ..., soussigné,

A reçu en la forme authentique le présent acte authentique à la requête de ...

Tożsamość stawających notariusz ustalit na podstawie dowodów osobistych.

Kerbrat-Orecchioni (1997 : 40) remarque que les pronoms de la troisième personne, contrairement aux embrayeurs, montrent que l'individu dénoté n'est ni locuteur, ni allocutaire. Les signataires des actes, présentés à la troisième personne s'effacent en tant qu'auteurs et destinateurs des énoncés, ils deviennent seulement l'objet des décisions. L'utilisation de la troisième personne peut être considérée comme la marque de l'intervention du législateur car les stipulations se font dans le respect de la loi.

3.2. Dans le corpus, on constate l'utilisation des formes impersonnelles du verbe. L'impersonnalisation peut être définie de la façon suivante : «elle décrit une action (...) comme si aucun sujet ou agent n'avait la responsabilité de cette action ou de cette modalisation » (Charaudeau, 1992 : 314). Dans de nombreux actes juridiques français, on trouve l'emploi du il impersonnel à propos duquel E. Benveniste constate qu'il peut être «une infinité de sujets ou aucun» $(1966: 230)$ :

Il a été arrêté et convenu ce qui suit...

Il a été procédé à la rédaction de l'acte ... 
La forme impersonnelle permet de ne pas expliciter le sujet des phrases, les locuteurs; il remplace le pronom indéfini on, qui n'est pas employé dans ce genre.

L'emploi de la forme impersonnelle donne aussi la possibilité d'amalgamer l'instance du locuteur et celle de l'allocutaire de l'énoncé :

Il peut être décidé par les associés, dans ce délai, de procéder à la dissolution de la société.

Il est établi une feuille de présences, émargée par chaque membre de l'assemblée.

Les agents des actions s'effacent comme si l'événement s'élaborait d'une manière autonome. "L'agent de l'action disparait, ce qui a pour effet de transformer l'action en un événement qui se produit par lui-même sans qu'aucun actant soit concerné, du même coup le contenu sémantique du verbe devient plus dense » (Charaudeau, $1992: 146)$ :

Il est formé entre les soussignés une société anonyme.

Il est tenu une comptabilité régulière des opérations sociales conformément à la loi.

De plus, dans tous les exemples cités, on constate la forme impersonnelle du passif.

M. Wojtak mentionne les formes impersonnelles des verbes comme des traits caractéristiques du style des écrits officiels polonais (2012: 160-161). Les actions ordonnées sont exprimées par des verbes à la troisième personne du singulier accompagnés du pronom się :

Walne Zgromadzenie zwoluje się przez dwukrotne ogloszenie w prasie lokalnej.

Les formes impersonnelles avec les suffixes -(o)no, -ano, -to sont caractéristiques des clôtures ou des préambules des actes notariés, dans lesquels celui qui accomplit les actions n'est pas nommé :

Umowę sporzadzono...

Pobrano tytułem opłaty notarialnej...

Akt ten stawajacym odczytano, przyjęto i podpisano.

La voix impersonnelle produit les mêmes effets dans les documents polonais et français. Elle contribue à créer le ton neutre et impersonnel de ces textes. Elle marque le caractère impersonnel de la règle en la présentant comme une réalité objective (Cornu, 2000 : 280). Avant tout, elle efface l'agent de l'action, la source des décisions, ce qui les rend incontestables. Tout cela tend à «donner une impression d'objectivité par un détachement impersonnel, artificiel, entre l'auteur du message et le récepteur » (Didier, $1987: 9)$. 
3.3. Dans les textes juridiques français et polonais, l'usage de la voix passive est très courant.

Le siège social est fixé à...

Umowa zostaje zawarta na czas...

La voix passive permet de recentrer le propos sur l'objet de l'action; en revanche l'agent n'est pas explicité dans ces phrases. De ce fait, l'action, la décision, apparaît comme objective et irrévocable, émanant d'une autorité incontestable.

Nombreuses sont aussi les phrases où l'agent est exprimé par un complément. Le patient, c'est-à-dire l'actant non humain ou humain qui subit l'action (Charaudeau, 1992: 383), est le sujet de la phrase. L'agent se trouve dans une position rhématique, ce qui attire davantage l'attention sur lui. Dans ces exemples, l'information porte sur l'autorité ou l'institution ayant le droit d'agir, c'est-à-dire de prendre des décisions, de diriger, etc.

L'assemblée est présidée par un gérant.

Wspólnicy moga być reprezentowani na Zgromadzeniu Wspólników przez osoby mające petna zdolność do czynności prawnych.

Dans les textes français, on trouve deux formes de passif, caractéristiques du style juridique : le passif initial et le passif impersonnel :

Est désigné comme représentant Monsieur...

Il est décidé qu'aucune rémunération ne sera versée à la gérante.

Les phrases à la troisième personne à la voix passive sont fréquentes dans les textes en français et en polonais. Toutefois, les verbes impersonnels à voix passive construits avec le verbe être impersonnel suivi d'un participe passé sont propres au français. En polonais ils trouvent leurs équivalents dans les verbes impersonnels avec les suffixes -no, -to, ou les verbes à la forme nominale en fonction de passif.

La convention qui consiste à utiliser la non-personne, les tournures impersonnelles et le passif s'applique à la rédaction des textes juridiques français et polonais. Elle souligne qu'il ne s'agit pas de rapports entre les personnes mais plutôt entre les fonctions : entre le bailleur et le locataire, entre les associés, entre le prêteur et l'emprunteur, etc. Elle rend le texte officiel, et produit un effet de généralisation. L'emploi de la troisième personne et des tournures impersonnelles permet également de ne pas nommer les auteurs des stipulations et, de ce fait, de renforcer leur caractère injonctif. Les voix passive et impersonnelle insistent sur le procès, sur ce qui est ordonné, interdit, indispensable pour obtenir 
un certain but, etc. En employant ces formes, les locuteurs se donnent de l'importance. Ils empruntent le style du législateur et signalent qu'ils agissent au nom de la loi.

Chacune des parties au contrat est à la fois l'émetteur et le récepteur du texte, les clauses sont le fruit de la rencontre des volontés et elles obligent les deux parties. Cependant, dans le cas des actes notariés, c'est le notaire qui rapporte la volonté des comparants. De plus, les contrats sont rédigés dans le respect de la loi, et la voix du législateur y est fort présente. Les contractants sont aussi les destinataires des normes inclues dans les textes de droit qui régissent les relations en question.

\section{COMPARAISON DES MOYENS D'EXPRESSION DU CARACTÈRE PERFORMATIF DES CONTRATS FRANÇAIS ET POLONAIS}

D'après C. Boquet (2008), les contrats relèvent du mode performatif dans la mesure où ces énoncés créent la réalité, et il remarque que les techniques d'expression de ce mode de discours varient d'une langue à l'autre. Les modalités énonciatives dominantes de ces textes sont, en reprenant les termes de P. Charaudeau (1992), l'injonction, l'obligation, l'autorisation. L'injonction est une modalité de l'acte allocutif, le locuteur formule une action à réaliser en l'imposant d'une manière comminatoire, pour que l'interlocuteur l'effectue. Il se donne donc le statut d'autorité. Quant à l'interlocuteur, il est supposé compétent pour exécuter l'injonction. Il reçoit une obligation à laquelle il est censé se soumettre. S'il refuse, il risque d'être sanctionné (P. Charaudeau, 1992 : 582). L'interdiction est considérée comme une variante de l'injonction avec cette particularité que c'est une non-exécution qui est imposée à l'interlocuteur. L'autorisation est un acte d'énonciation par lequel le locuteur donne à l'interlocuteur le droit d'exécuter une action et se donne le statut de garant de ce droit. Enfin, l'obligation est une modalité de l'acte élocutif par lequel le locuteur déclare devoir accomplir une action (Charaudeau, 1992 : 575). Il peut s'y obliger lui-même ou bien se soumettre à un ordre émanant d'une autorité. Dans les textes étudiés, nous trouvons l'injonction dans les passages où le législateur oblige des sujets de droit, ou bien dans ceux où un intervenant à l'acte oblige l'autre à faire ou à ne pas faire quelque chose. De même, les actes d'autorisation sont présents dans les fragments de texte où une autorité ou une partie au contrat donne à l'autre partie la possibilité de bénéficier d'un droit. L'obligation est exprimée dans les actes dans lesquels les signataires s'obligent eux-mêmes à faire ou à ne pas faire quelque chose. Dans notre travail, nous parlerons de contrainte pour désigner ces trois modalités. 
4.1. Les documents que nous étudions portent des marques lexicales et syntaxiques de la contrainte telles que les verbes et autres mots performatifs. Ainsi, les documents étudiés abondent en verbes qui explicitent et précisent le caractère de la prise de parole des signataires. Ce sont les verbes nommés par Austin (1991) expositifs : affirmer, attester, certifier, confirmer, déclarer, exposer, reconnaître, oświadczać, zaświadczać, potwierdzać.

Les soussignés (...) agissant en qualité de seuls membres, tous deux gérants, de ladite société, déclarent ce qui suit...

Stawajacy oświadczają, że...

Le notaire soussigné certifie et atteste...

Sprzedajacy niniejszym deklaruje, gwarantuje i uzgadnia, co następuje...

Les signataires des contrats s'engagent à faire ou à ne pas faire une chose, se chargent de remplir un devoir, etc. Ils accomplissent alors, selon la terminologie de Searle (1982), un acte promissif. Les verbes promissifs sont employés dans les documents polonais et français, ce sont des verbes tels que s'obliger, s'engager, se charger, zobowiazać się, podjać się :

L'emprunteur s'oblige à entretenir, à ses frais, le bien prêté.

Bioracy do używania zobowiazuje się do dokonania wszelkich napraw.

Dans les textes analysés, on trouve des obligations, des autorisations ou des interdictions d'exécuter des faits, adressées aux signataires ou à d'autres personnes. Ce sont des déclarations par lesquelles ces locuteurs exercent un pouvoir; il s'agit donc de déclarations dans le sens de Searle. Elles sont formulées à l'aide de verbes appelés par Austin exercitifs, tels que nommer, révoquer, annuler, donner, créer, mianować, dawać, tworzyć etc. :

Il a été constitué une société à responsabilité limitée.

Stawajacy oświadczaja, że zawiazuja jako zatożyciele spótkę cywilna zwanq dalej , spótka".

Dans les exemples cités ci-dessus, les phrases polonaises sont plus explicites du fait de l'emploi de l'expositif oświadczać (déclarer).

Parmi les verbes exercitifs il y a également des verbes exprimant l'injonction et l'autorisation, tels que charger, obliger, tenir. Dans ces textes, nombreux sont les énoncés exercitifs qui autorisent quelqu'un à faire quelque chose, ils contiennent les verbes et les syntagmes suivants : autoriser, accorder le droit, libérer des prétentions, conférer des pouvoirs, zezwalać, upoważniać, przyznać prawo.

Le propriétaire accorde le droit au locataire d'exploiter, d'utiliser les équipements loués.

Wynajmujacy zezwala najemcy na wykonanie na wlasny koszt odświeżenia domu. 
On emploie également des verbes comme convenir, arrêter, établir, ustalać, uzgadniać, etc. Voici les formules introduisant les stipulations des contrats :

Il a été convenu ce qui suit...

M... M.... et Mme... ont établi ainsi qu'il suit, les statuts d'une société civile.

Strony ustalaja nastepujace warunki umowy...

Les inventaires des verbes performatifs employés pour la rédaction des textes en français et en polonais sont similaires. Le caractère performatif est souligné aussi par l'adverbe niniejszym et les locutions adverbiales par la présente et par les présentes, de même que les adjectifs niniejszy et présent qui font partie de la déixis :

Il est formé par les présentes une société...

Niniejszym, oświadczam, że

Mis à part les verbes performatifs, l'injonction est exprimée par des moyens lexicaux tels que le verbe modal devoir pour les textes français. Dans les documents polonais, le verbe modal musieć n'est pas fréquent. En revanche, on emploie les verbes wymagać, powinien si le sujet est inanimé :

Pelnomocnictwo wymaga formy pisemnej pod rygorem nieważności.

Protokoly winny być zebrane w księgę protokołów.

Pour les sujets animés, on trouve les verbes : powinien, winien. Ceci est conforme aux règles de rédaction législative (Wronkowska \& Zieliński, 2012) parce que comme l'a remarqué M. Zieliński, le langage de droit, celui du législateur, influe sur le langage de la pratique juridique non judiciaire (Zieliński, 1999 : 66). L'idée de contrainte est également traduite par le verbe obowiazywać, ponosić odpowiedzialność et le verbe impersonnel należy.

Dans les documents des deux langues, l'obligation est souvent exprimée par les verbes à la voix passive. Dans les textes français, on trouve les formes être tenu, être chargé, être obligé et le verbe être suivi d'un adjectif : être responsable. Dans les textes parallèles polonais on trouve les constructions być + imiestów bierny, par exemple być zobowiazanym, być obowiazanym :

Le débiteur sera tenu de rembourser tous les frais engagés pour ce procès.

Za opóźnienie w zwrocie pożyczki Pożyczkobiorca zobowiazany jest do zapłaty odsetek za zwtokę.

L'idée d'injonction est aussi exprimée par d'autres moyens lexicaux : le substantif une obligation, l'adverbe obligatoirement, le substantif obowiazek dans les documents polonais. 
Les décisions sont parfois accompagnées d'informations sur des sanctions au cas où le droit ne serait pas respecté. Le rappel explicite de la sanction renforce le caractère contraignant des documents.

4.2. Ce qui est caractéristique des textes juridiques de mode performatif, c'est que dans de nombreux cas, les actes de langage ne portent pas de marques ostensibles. Les énonciateurs accomplissent des actes sans employer de verbes performatifs. Ainsi, des phrases comme celle-ci, tirée d'un contrat de prêt à usage, peuvent être traitées comme des performatifs primaires :

Le prêteur tiendra le bien prêté à la disposition de l'emprunteur.

Biorący do używania będzie używat lokalu zgodnie z jego przeznaczeniem.

Austin (1991) a remarqué que les performatifs peuvent se prêter à plusieurs manipulations sans perdre leur performativité. Il cite à titre d'exemple l'ellipse, la transformation passive ou impersonnelle et l'addition d'un modalisateur ou d'un préfixe. Les contrats français et polonais sont rédigés surtout à l'indicatif et les temps dominants sont le présent et le futur. Le présent actuel (Charaudeau, 1992 : 459) renforce le caractère performatif des énoncés.

Dans certains contextes où les actes anticipent les comportements des sujets de droit dans des conditions précises, nous pouvons distinguer un présent imaginé, dont voici la définition donnée par P. Charaudeau (1992: 453) : «Le processus n'est envisagé qu'à certaines conditions, dans un moment imaginé ».

Comme illustration, prenons d'abord une phrase tirée des statuts d'une société civile où l'on définit les conditions de cession de parts sociales par l'un des associés :

En cas d'agrément, la cession doit être régularisée dans le délai prévu au § II, alinéa $1^{e r}$, ci-dessus.

On retrouve cet emploi du présent dans les documents polonais :

W przypadku niedokonania zwrotu pożyczki w ustalonym terminie, Pożyczkobiorca obowiazany jest do zaptaty odsetek za zwtokę...

Dans ces exemples, le présent a un caractère à la fois imaginé et injonctif.

La valeur modale dominante du présent dans les textes de droit est celle d'obligation ou d'injonction. Il ne décrit pas ce qui est mais plutôt ce qui doit être.

Prenons à titre d'exemple des fragments de statuts d'une société civile, français et polonais :

...chaque associé est tenu des dettes sociales indéfiniment dans la proportion de sa part...

Za zobowiqzania spótki wobec osób trzecich odpowiadaja wspólnicy... 
L'indicatif présent à valeur générique ou imaginé remplace, dans les textes juridiques, l'impératif. Il contribue à exprimer l'injonction ou l'obligation. Il atténue l'ordre tout en rendant le devoir inévitable. G. Cornu (2000) analyse les effets que produit l'emploi du présent dans les textes législatifs :

Psychologiquement, l'indicatif présent offre d'ailleurs des avantages. Il occulte celui qui donne l'ordre et ne brandit pas le pouvoir d'ordonner. C'est une façon plus discrète, plus douce et plus diplomatique de commander. La référence à ce qui est pourrait même faire imaginer que la règle énoncée n'est pas arbitrairement imposée, mais naturellement fondée, que le droit est proche de la nature des choses. Dans la même voie, la référence à ce qui se passe montre le sujet de droit agissant, dans le respect de la loi, conformément à la règle, transformant en somme le droit en fait, traduisant la règle dans la réalité (Cornu, $2000: 272$ ).

C. Boquet souligne que la possiblité d'employer l'indicatif pour exprimer des ordres est la conséquence du caractère performatif du discours législatif.

La fonction injonctive du temps présent ainsi que du futur est également démontrée par des chercheurs polonais. Z. Ziembiński et M. Zieliński (1992) observent que même si, dans la langue polonaise standard, la fonction directive des phrases à l'indicatif n'est que secondaire, elle devient primordiale dans les textes juridiques du fait du caractère normatif de ces documents.

Dans les exemples français et polonais cités, l'indicatif ne sert pas à décrire une norme, informer sur une obligation ou une interdiction mais, à accomplir l'acte d'ordonner, d'interdire, etc. (Zieliński, 1999 : 61, Boquet, $2008: 24$ ).

Les exemples ci-dessous montrent cette fonction du futur :

Koszty zawarcia umowy ponosza strony po połowie.

Les loyers seront payables d'avance le premier jour ouvré de chaque mois par virement bancaire...

Czynsz będzie placony z góry...

Le preneur rendra la parcelle en fin de bail en bon état d'engrais et d'entretien.

Quant aux textes polonais, la plupart des verbes qui y sont employés au futur sont des verbes perfectifs, plus propres à exprimer un ordre et dont l'interprétation modale permet de prendre en considération la totalité de l'action ainsi que son résultat (Tomaszkiewicz, $1988: 122$ ) :

Koszty umowy poniesie...

Zleceniodawca opracuje $i$ zatwierdzi u kompetentnych władz niezbędna dokumentacje techniczna. Zleceniobiorca dostarczy materiaty konstrukcyjne, ustawi i uzupetni budowle, usunie wszelkie usterki $i$ wykona prace na zewnatrz.

On peut constater que les manières de formuler la contrainte sont semblables dans les textes français et polonais, bien qu'il existe quelques différences. En ce 
qui concerne les verbes performatifs, l'expositif oświadczać est employé plus souvent que déclarer en français parce qu'il accompagne les verbes exercitifs. Le caractère performatif est donc plus explicite dans la version polonaise. De plus, le polonais préfère les verbes perfectifs, plus aptes à exprimer l'ordre.

Le caractère dispositif des actes juridiques est renforcé par le fait que l'on ne trouve, ni dans la version polonaise, ni dans la française, d'argumentation, de tentative de justifier les décisions. Tomasz Gizbert-Studnicki (1986 : 59-60) remarque que le législateur énonce les dispositions sans en chercher l'approbation et, par conséquent, sans en présenter les raisons. C'est pourquoi on ne trouve pas dans les textes analysés de conjonctions de cause ni de conséquence.

Pour clôturer notre réflexion sur les marques lexicales de la contrainte dans les actes juridiques, nous rapportons l'opinion de M. Wojtak (2012) selon laquelle le répertoire des formules exprimant la directive dans les textes officiels est limité parce que ces tournures sont stéréotypées. Même si les moyens d'exprimer la contrainte que nous avons cités ne forment pas une liste exhaustive, nous pouvons conclure d'après notre analyse que leur choix est assez limité. Bien que les formules de contrainte apparaissent souvent dans les textes juridiques, leur éventail n'est pas large. La répétition de la même expression dans plusieurs documents renforce le caractère conventionnel de ceux-ci. Ces formules stéréotypées ne traduisent pas toujours des réalités propres au droit, mais elles font partie du discours juridique.

\section{EN GUISE DE CONCLUSION}

Nous avons comparé les relations intersubjectives dans les contrats français et polonais, et les moyens de les exprimer. La comparaison du caractère performatif a relevé des ressemblances et a permis de recueillir des manières de l'exprimer par des moyens lexicaux et syntaxiques propres à chacun des discours en question. Ainsi, les sujets de la communication sont exprimés à la troisième personne. En ce qui concerne l'impersonnalité, qui est caractéristique aussi bien des documents français que polonais, elle est formulée en français à l'aide du pronom impersonnnel $i l$, préféré au pronom on, mais aussi à l'aide du passif impersonnel et initial; dans les écrits polonais, outre la voix passive, on constate l'emploi des formes impersonnelles avec les suffixes -no et -to et l'usage des formes pronominales des verbes en fonction de passif. Etant donné l'appartenance des contrats au genre des textes juridiques de mode performatif, on y constate des modalités d'énonciation telles que l'injonction, l'obligation et l'autorisation. Le répertoire des moyens d'expression lexicaux est relativement restreint dans chacun des discours juridiques, ce qui résulte de l'usage et de 
l'influence du discours des textes législatifs. De même, du fait que les contrats font partie d'un polysystème génologique plus large, ces modalités d'énonciation sont exprimées indirectement au moyen de l'indicatif.

L'étude des relations hiérarchiques dans ce genre de texte devrait aussi être complétée par une analyse des marques de la portée générale des normes de droit, telles que les adjectifs et les pronoms indéfinis à valeur d'exhaustivité, de distribution et de discrimination, ainsi que les négatifs. L'autorité de la loi se manifeste aussi par des renvois explicites aux textes normatifs émanant du législateur et par le souci de la cohésion interne des textes. Ils sont réalisés par des moyens lexicaux spécifiques dont la comparaison peut fournir au traducteur des outils bien précis.

En effet, la comparaison des genres permet de produire un texte qui est l'effet de négociations entre deux polysystèmes discursifs différents (Walkiewicz, 2012 : 71). Même si le traducteur ne peut pas adopter entièrement le genre de la culture d'arrivée, car la structure, la mise en page du document juridique sont porteurs de sens, et que les termes du texte de départ ne trouvent pas toujours d'équivalent dans le système du droit d'arrivée, la comparaison des genres stéréotypés tels que les contrats peut donner des solutions au niveau micro, celui des formules conventionnelles spécifiques des genres de texte et du discours juridique en question. Dans le présent article nous avons comparé les moyens d'expression des relations entre les sujets de la communication, ainsi que les expressions du caractère performatif des textes afin de donner des solutions pratiques applicables lors du processus de la traduction. Ceci permet d'obtenir une traduction idiomatique, respectant les règles et les conventions fixées dans la langue et la culture d'arrivée, et de prendre pour modèle la façon spontanée de s'exprimer des usagers du discours d'arrivée (Kierzkowska, 2002). 


\section{BIBLIOGRAPHIE}

Antoniewska, D. (2005). L'analyse contrastive des textes modèles de droit civil français et polonais appliquée à la traduction (Unpublished doctoral dissertation). Adam Mickiewicz University in Poznań, Poland.

Austin, J.L. (1991). Quad dire, c'est faire (G. Lane, Trans.). Paris : Editions du Seuil. (Original work published $1962 \mathrm{r}$.)

Benveniste, É. (1966). Problèmes de linguistique générale. Paris : Éditions Gallimard.

Boquet, C. (2008). La traduction juridique : fondement et méthode. Bruxelles : De Boeck.

Charaudeau, P. (1992). La grammaire du sens et de l'expression. Paris : Hachette Education.

Code civil-2013. (2012). Éditions Prat.

Cornu, G. (2000). Linguistique juridique. ( $2^{\text {nd }}$ ed.) Paris : Montchrestein.

Didier, E. (1987). Le langage du droit et la nouvelle traduction juridique. L'actualité terminologique, 20 (5), 7-11.

Fontaine, M., Cavalerie, R., Hassenforder, J.-A. (2000). Dictionnaire de droit (2nd ed.). Paris : Foucher.

Gémar, J.-C. (1995). Traduire ou l'art d'interpréter. Langue, droit et société : éléments de jurilinguistique, tome 2: Application. Traduire le texte juridique. Québec : Presses de l'Université du Québec.

Gizbert-Studnicki, T. (1986). Język prawny z perspektywy socjolingwistycznej. Warszawa - Kraków : Państwowe Wydawnictwo Naukowe.

Grzmil-Tylutki, H. (2007). Gatunek w świetle francuskiej teorii dyskursu. Kraków : Universitas.

Guillien, R. \& Vincent, J. (2001). Lexique des termes juridiques (13th ed.). Paris : Dalloz.

Jopek-Bosiacka, A. (2006). Przektad prawny i sadowy. Warszawa : Wydawnictwo Naukowe PWN.

Kerbrat-Orecchioni, C. (1997). L'énonciation. De la subjectivité dans le langage. Paris : Armand Colin.

Kierzkowska, D. ( 2002). Ttumaczenie prawnicze. Warszawa : Wydawnictwo Tepis.

Radwański, Z. (Eds.), (2007). Prawo cywilne - część ogólna (9th ed.). Warszawa : C.H. Beck : Instytut Nauk Prawnych PAN.

Searle, J.R. (1982). Sens et expression (J. Proust, Trans.). Paris : Minuit. (Original work published 1979 r.)

Solova, R. (2013). Norma i praktyka w przekładzie tekstów skonwencjonalizowanych. Wrocław : Oficyna Wydawnicza ATUT.

Tomaszkiewicz, T. (1988). Étude comparative de quelques indices du concept d'énonciation en français et en polonais. Poznań : Wydawnictwo Naukowe UAM.

Walkiewicz, B. (2012). La traduction en tant que discours. Studia Romanica Posnaniensia, XXXIX, 71-86.

Walkiewicz, B. (2013). Architektura tekstów o architekturze. Poznań : Wydawnictwo Naukowe UAM.

Wojtak, M. (2012). Styl urzędowy. In J. Bartmiński (Eds.), Wspótczesny język polski (4th ed.). (pp. 155-171). Lublin : Wydawnictwo Uniwersytetu Marii Curie-Skłodowskiej.

Wronkowska, S. \& Zieliński, M. (2012). Komentarz do zasad techniki prawodawczej z dnia 20 czerwca 2002 (2nd ed.). Warszawa : Wydawnictwo Sejmowe.

Ziembiński, Z. \& Zieliński, M. (1992). Dyrektywy i sposób ich wypowiadania. Warszawa : Polskie Towarzystwo Semiotyczne.

Zieliński, M. (1999). Języki prawne i prawnicze. In W. Pisarek, Polszczyzna 2000. Orędzie o stanie języka na przełomie tysiącleci (pp. 50-74). Kraków : Ośrodek Badań Prasoznawczych Uniwersytet Jagielloński. 